\title{
FIXED POINTS AND VARIATIONAL PRINCIPLE WITH APPLICATIONS TO EQUILIBRIUM PROBLEMS ON CONE METRIC SPACES
}

\author{
Jong-Sook BaE And SeOng-Hoon Cho
}

\begin{abstract}
The aim of this paper is to establish variational principle on cone metric spaces and to give some existence theorems of solutions for equilibrium problems on cone metric spaces. We give some equivalences of an existence theorem of solutions for equilibrium problems on cone metric spaces.
\end{abstract}

\section{Introduction}

In $[11,12]$, the author obtained a variational principle, so called Ekeland's variational principle. It is one of the most important results obtained in nonlinear analysis, and is useful tools to solve problems in optimization, optimal control theory, game theory, nonlinear equations and dynamical systems $[5,6,10,12,13,30]$.

It was known that Petal's theorem, Daneš's drop theorem, KrasnoselskiiZabrjeko and Caristi's fixed point theorem are equivalent to Ekeland's variational principle (see [25] and references therein). Since this variational principle was proved, there have appeared many extensions and equivalence formulations of Ekeland's variational principle (see [1, 22, 24, 29]).

Recently, the author [14] introduced the notion of cone metric spaces as a generalization of metric spaces. They introduced the concept of convergence in cone metric spaces and obtained some fixed point theorems for contractive mappings defined on cone metric spaces.

In this paper, we obtain an extension of Ekeland's variational principle to cone metric spaces. We present Caristi's fixed point theorem, Takahashi's minimization theorem and an equilibrium version of Ekeland's variational principle

Received December 5, 2011; Revised March 5, 2012.

2010 Mathematics Subject Classification. 47H10, 54H25.

Key words and phrases. variational principle, equilibrium problem, upper semi-continuous, cone metric space.

The author(S. H. Cho) was supported by Basic Science Research Program through the National Research Foundation of Korea(NRF) funded by the Ministry of Education, Science and Technology(No. 2011-0012118). 
in the setting of complete cone metric spaces. And then we prove that these results and Ekeland's variational principle are equivalent. We establish some existence theorems of solution for equilibrium problem on cone metric spaces without any convexity assumption. And then, we obtain some equivalences of an existence theorem of solution for equilibrium problem on cone metric spaces.

\section{Preliminaries}

Consistent with Huang and Zhang [14], the following definitions will be needed in the sequel.

Let $E$ be a topological vector space. A subset $P$ of $E$ is a cone if the following conditions are satisfied:

(i) $P$ is non-empty closed and $P \neq\{0\}$,

(ii) $a x+b y \in P$, whenever $x, y \in P$ and $a, b \in \mathbb{R}(a, b \geq 0)$,

(iii) $P \cap(-P)=\{0\}$.

Given a cone $P \subset E$, we define a partial ordering $\leq$ on $E$ with respect to $P$ by $x \leq y$ if and only if $y-x \in P$. We write $x<y$ to indicate that $x \leq y$ but $x \neq y$.

For $x, y \in E, x \ll y$ stand for $y-x \in \operatorname{int}(P)$, where $\operatorname{int}(P)$ is the interior of $P$. A cone $P$ is called regular if every increasing sequence which is bounded from above is convergent. That is, if $\left\{u_{n}\right\}$ is a sequence such that for some $z \in E$

$$
u_{1} \leq u_{2} \leq \cdots \leq z,
$$

then there exists $u \in E$ such that

$$
\lim _{n \rightarrow \infty} u_{n}=u .
$$

Equivalently, a cone $P$ is regular if and only if every decreasing sequence which is bounded from below is convergent.

If $E$ is a normed space, a cone $P$ is called normal whenever there exists a number $M>0$ such that for all $x, y \in E, 0 \leq x \leq y$ implies $\|x\| \leq M\|y\|$.

It is well known that every regular cone in a normed space is normal (see $[14,28])$.

A cone $P$ is complete if every upper bounded non-empty subset $A$ of $E$, $\sup A$ exists in $E$. Equivalently, a cone $P$ is complete if every lower bounded non-empty subset $A$ of $E, \inf A$ exists in $E$.

If $E$ is a normed space, a complete cone $P$ is continuous whenever, for any upper bounded chain $\left\{x_{\alpha}: \alpha \in \Gamma\right\}, \sup \left\{\left\|x_{\alpha}-\sup \left\{x_{\beta}: \beta \in \Gamma\right\}\right\|: \alpha \in \Gamma\right\}=0$.

Equivalently, if $E$ is a normed space, a complete cone $P$ is continuous whenever, for any lower bounded chain $\left\{x_{\alpha}: \alpha \in \Gamma\right\}, \inf \left\{\left\|x_{\alpha}-\inf \left\{x_{\beta}: \beta \in \Gamma\right\}\right\|\right.$ : $\alpha \in \Gamma\}=0$.

Note that if $E$ is a normed space and $P$ is complete and continuous, then for every non-increasing (resp. non-decreasing) sequence $\left\{x_{n}\right\} \subset P$ bounded from below (resp. above), we have $\lim _{n \rightarrow \infty} x_{n}=\inf _{n} x_{n}\left(\right.$ resp. $\lim _{n \rightarrow \infty} x_{n}=$ $\left.\sup _{n} x_{n}\right)$. 
The authors $[2,3,8,14,15,16,26,31,34]$ obtained fixed point theorems on cone metric spaces under assumption that the cone is normal. Also, the authors $[7,23,32]$ proved fixed point results under assumption that the cone is regular. And the authors $[4,9,17,18,19,21,20,27,28]$ do not use the notion of normality or regularity to obtain their results on cone metric spaces.

In this paper, we use the concept of regularity to obtain our results.

Without special mention, we assume that $E$ is a normed space, $P$ is a cone in $E$ with $\operatorname{int}(P) \neq \emptyset$ and $\leq$ is a partial ordering with respect to $P$. Also, we assume that $2^{E}$ (resp. $K(E)$ ) is the family of all non-empty (resp. non-empty compact) subsets of $E$.

For a non-empty set $X$, a mapping $d: X \times X \rightarrow E$ is called cone metric [14] on $X$ if the following conditions are satisfied:

(i) $0 \leq d(x, y)$ for all $x, y \in X$, and $d(x, y)=0$ if and only if $x=y$,

(ii) $d(x, y)=d(y, x)$ for all $x, y \in X$,

(iii) $d(x, y) \leq d(x, z)+d(z, y)$ for all $x, y, z \in X$.

Let $(X, d)$ be a cone metric space. A sequence $\left\{x_{n}\right\}$ of points in $X$ converges [14] to a point $x \in X$ (denoted by $\lim _{n \rightarrow \infty} x_{n}=x$ or $x_{n} \rightarrow x$ ) if for any $c \in \operatorname{int}(P)$, there exists $N \in \mathbb{N}$ such that for all $n>N, d\left(x_{n}, x\right) \ll c$. A sequence $\left\{x_{n}\right\}$ of points in $X$ is Cauchy [14] if for any $c \in \operatorname{int}(P)$, there exists $N \in \mathbb{N}$ such that for all $n, m>N, d\left(x_{n}, x_{m}\right) \ll c$. $(X, d)$ is called complete if every Cauchy sequence is convergent.

Note that if $\lim _{n \rightarrow \infty} d\left(x_{n}, x\right)=0$, then $\lim _{n \rightarrow \infty} x_{n}=x$. The converse is true if $E$ is a normed space and $P$ is a normal cone. Also, note that if $\lim _{n, m \rightarrow \infty} d\left(x_{n}, x_{m}\right)=0$, then $\left\{x_{n}\right\}$ is a Cauchy sequence in $X$. If $E$ is a normed space and $P$ is a normal cone, then $\left\{x_{n}\right\}$ is a Cauchy sequence in $X$ if and only if $\lim _{n, m \rightarrow \infty} d\left(x_{n}, x_{m}\right)=0$.

Let $(X, d)$ be a cone metric (or metric) space. A subset $A$ of $X$ is called sequentially closed if for any sequence $\left\{x_{n}\right\} \subset X$ with $\lim _{n \rightarrow \infty} x_{n}=x$, we have $x \in A$. A subset $A$ of $X$ is sequentially compact if every sequence in $X$ has a convergent subsequence. A mapping $g: X \rightarrow E$ is sequentially continuous at $x \in X$ if, for any sequence $\left\{x_{n}\right\} \subset X$ with $\lim _{n \rightarrow \infty} x_{n}=x$, we have $\lim _{n \rightarrow \infty} g x_{n}=g x$. A set-valued mapping $F: X \rightarrow 2^{E}$ is called bounded from below if there exists $z \in E$ such that $F x-z \subset P$ for all $x \in X$. A setvalued mapping $F: X \rightarrow 2^{E}$ is called sequentially lower semi-continuous at $x_{0} \in X$ if, for any closed subset $C$ of $E$ and for any sequence $\left\{x_{n}\right\} \subset X$ with $\lim _{n \rightarrow \infty} x_{n}=x_{0}$, there exists $N \in \mathbb{N}$ such that, for all $n>N, F x_{n} \not \subset C$ whenever $F x_{0} \not \subset C$.

When $F$ is sequentially lower semi-continuous at each point in $X$, we say that $F$ is sequentially lower semi-continuous.

A set-valued mapping $F: X \rightarrow 2^{E}$ is sequentially upper semi-continuous at $x_{0} \in X$ if, for any closed subset $C$ of $E$ and for any sequence $\left\{x_{n}\right\} \subset X$ with $\lim _{n \rightarrow \infty} x_{n}=x_{0}$, there exists $N \in \mathbb{N}$ such that, for all $n>N, F x_{n} \cap C=\emptyset$ whenever $F x_{0} \cap C=\emptyset$. 
We say that $F$ is sequentially upper semi-continuous if $F$ is sequentially upper semi-continuous at each point in $X$.

Example 2.1. Let $X=\mathbb{R}, E=\mathbb{R}^{2}$ and $P=\mathbb{R}_{+}^{2}$. We define a set-valued mapping $F: X \rightarrow 2^{E}$ by

$$
F x=\left\{\begin{array}{l}
\{(0,0)\}, \text { if } x=0 ; \\
\{(-|x|, 0),(0,-|x|)\}, \text { if } x \neq 0 .
\end{array}\right.
$$

Then $F$ is sequentially lower semi-continuous and sequentially upper semicontinuous.

We have the following properties of sequentially lower semi-continuous and sequentially upper semi-continuous, respectively.

Lemma 2.1. A set-valued mapping $F: X \rightarrow 2^{E}$ is sequentially lower semicontinuous if and only if the set $L(C)=\{x \in X: F x \subset C\}$ is sequentially closed for all closed subset $C$ of $E$.

Proof. Let $C$ be a closed subset of $E$. Suppose that $L(C)$ is sequentially closed. Let $x_{0} \in X$ be fixed, and let $\left\{x_{n}\right\}$ be any sequence of points in $X$ such that $\lim _{n \rightarrow \infty} x_{n}=x_{0}$. If $F x_{0} \not \subset C$, then $x_{0} \notin L(C)$. Since $L(C)$ is sequentially closed, there exists $N \in \mathbb{N}$ such that $x_{n} \notin L(C)$ for all $n>N$. Thus, $F x_{n} \not \subset C$ for all $n>N$. Hence $F$ is sequentially lower semi-continuous on $X$.

Assume that $F$ is sequentially lower semi-continuous on $X$. Let $C$ be any fixed closed subset of $E$, and let $\left\{x_{n}\right\}$ be a sequence of points in $L(C)$ such that $\lim _{n \rightarrow \infty} x_{n}=x_{0}$. If $x_{0} \notin L(C)$, then $F x_{0} \not \subset C$. Since $F$ is sequentially lower semi-continuous at $x_{0}$, there exists $N \in \mathbb{N}$ such that $F x_{n} \not \subset C$ for all $n>N$. Thus, $x_{n} \notin L(C)$ for all $n>N$, which is a contradiction. Hence $x_{0} \in L(C)$, and hence $L(C)$ is sequentially closed.

Lemma 2.2. A set-valued mapping $F: X \rightarrow 2^{E}$ is sequentially upper semicontinuous if and only if the set $U(C)=\{x \in X: F x \cap C \neq \emptyset\}$ is sequentially closed for all closed subset $C$ of $E$.

Proof. The proof is similar as proof of Lemma 2.1.

Lemma 2.3. If a set valued mapping $F: X \rightarrow 2^{E}$ is sequentially lower semicontinuous and a mapping $g: X \rightarrow E$ is sequentially continuous, then $F+g$ is sequentially lower semi-continuous.

Proof. Suppose that $F+g$ is not sequentially lower semi-continuous. Then there exists a closed subset $C$ of $E$ such that $L(C)=\{x \in X: F x+g x \subset C\}$ is not sequentially closed. Then there exists a sequence $\left\{x_{n}\right\} \subset L(C)$ with $\lim _{n \rightarrow \infty} x_{n}=z$ such that $z \notin L(C)$.

Since $F z \not \subset-g z+C$, there exists $y \in F z$ such that $y \notin-g z+C$. Then there exists $\epsilon>0$ such that

$$
B(y, \epsilon) \cap(-g z+C)=\emptyset,
$$


where $B(y, \epsilon)=\{x \in X:\|x-y\|<\epsilon\}$.

Since $F z \not \subset E-B\left(y, \frac{\epsilon}{2}\right)$ and $F$ is sequentially lower semi-continuous and $g$ is sequentially continuous, there exists $N \in \mathbb{N}$ such that, for all $n>N$,

$$
F x_{n} \cap B\left(y, \frac{\epsilon}{2}\right) \neq \emptyset
$$

and

$$
\left\|g x_{n}-g z\right\|<\frac{\epsilon}{2} .
$$

If $F x_{n} \subset-g x_{n}+C$ for some $n>N$, then there exists $y_{1} \in F x_{n} \cap B\left(y, \frac{\epsilon}{2}\right)$ such that $y_{1}=-g x_{n}+c_{1}$ for some $c_{1} \in C$, and $\left\|c_{1}-y_{1}-g z\right\|<\frac{\epsilon}{2}$. Thus we have $\left\|y-\left(-g z+c_{1}\right)\right\| \leq\left\|y-y_{1}\right\|+\left\|y_{1}+g z-c_{1}\right\|<\epsilon$. Hence $-g z+c_{1} \in B\left(y, \frac{\epsilon}{2}\right)$, which is a contradiction, because $B(y, \epsilon) \cap(-g z+C)=\emptyset$. Thus $F x_{n} \not \subset-g x_{n}+C$ for all $n>N$, and so $x_{n} \notin L(C)$ for all $n>N$, which is a contradiction.

For $A \subset E$ and $x \in E$, we denote $\rho(x, A)=\inf \{\|x-y\|: y \in A\}$.

Lemma 2.4. If a set valued mapping $F: X \rightarrow K(E)$ is sequentially upper semi-continuous and a mapping $g: X \rightarrow E$ is sequentially continuous, then $F+g$ is sequentially upper semi-continuous.

Proof. Suppose that $F+g$ is not sequentially upper semi-continuous. Then there exists a closed subset $C$ of $E$ such that $U(C)=\{x \in X: F x+g x \cap C \neq \emptyset\}$ is not sequentially closed. Then there exists a sequence $\left\{x_{n}\right\} \subset U(C)$ with $\lim _{n \rightarrow \infty} x_{n}=z$ such that $z \notin U(C)$. Thus, $F z \cap(-g z+C)=\emptyset$.

Let $\epsilon=\inf \{\rho(x,-g z+C): x \in F z\}$. Then since $F z$ is compact and $-g z+C$ is closed, we have $\epsilon>0$. Let $V=\left\{x \in E: \rho(x, F z)<\frac{1}{2} \epsilon\right\}$. Then

$$
V \cap(-g z+C)=\emptyset
$$

and

$$
F z \cap(E-V)=\emptyset .
$$

Since $F$ is sequentially upper semi-continuous and $g$ is sequentially continuous, there exists $N \in \mathbb{N}$ such that, for all $n>N$,

$$
F x_{n} \subset V
$$

and

$$
\left\|g x_{n}-g z\right\|<\frac{1}{2} \epsilon .
$$

If $F x_{n}+g x_{n} \cap C \neq \emptyset$ for some $n>N$, then there exists $u \in F x_{n}$ such that $u+g x_{n}=c_{1}$ for some $c_{1} \in C$. Hence $u=-g x_{n}+c_{1} \in V$. Thus we have

$$
\rho\left(-g z+c_{1}, F z\right) \leq\left\|-g z+c_{1}-u\right\|+\rho(u, F z)=\left\|g x_{n}-g z\right\|+\rho(u, F z)<\epsilon,
$$

which is a contradiction.

Hence $F x_{n}+g x_{n} \cap C=\emptyset$ for all $n>N$, and hence $\left\{x_{n}\right\}_{n>N} \not \subset U(C)$, which is a contradiction.

Lemma $2.5([14])$. Let $(X, d)$ be a cone metric space such that $P$ is normal. Then, for each $x \in X, d(x, \cdot)$ is sequentially continuous. 


\section{Fixed point theorems}

In this section, we obtain a fixed point theorem for set-valued mappings, which is closely related with the Ekeland's variational principle.

Lemma 3.1. Let $(X, d)$ be a cone metric space, and let $\left\{x_{n}\right\}$ be a sequence in $X$ such that $\lim _{n \rightarrow \infty} x_{n}=\bar{x} \in X$. Suppose that a set valued mapping $T: X \rightarrow 2^{X}$ satisfies the following conditions:

(1) $x_{n+1} \in T x_{n}$ and $T x_{n+1} \subset T x_{n}$ for all $n \geq 1$;

(2) $\lim _{n \rightarrow \infty} \sup \left\{\left\|d\left(x_{n}, u\right)\right\|: u \in T x_{n}\right\}=0$;

(3) $\bar{x} \in T x_{n}$ for all $n \geq 1$.

Then $\bigcap_{n \geq 1} T x_{n}=\{\bar{x}\}$.

If, in addition

(4) $T \bar{x} \neq \emptyset$ and $T \bar{x} \subset T x_{n}$ for all $n \geq 1$, then $T \bar{x}=\{\bar{x}\}$.

Proof. From (3), we have $\bar{x} \in \bigcap_{n \geq 1} T x_{n}$. If $u \in \bigcap_{n \geq 1} T x_{n}$, then

$$
\lim _{n \rightarrow \infty}\left\|d\left(x_{n}, u\right)\right\|=0
$$

by (2). Hence $\lim _{n \rightarrow \infty} d\left(x_{n}, u\right)=0$, and hence $\lim _{n \rightarrow \infty} x_{n}=u$. Thus $\bar{x}=u$, and so $\bigcap_{n \geq 1} T x_{n}=\{\bar{x}\}$.

Suppose that (4) is satisfied. Then $T \bar{x} \subset \bigcap_{n \geq 1} T x_{n}=\{\bar{x}\}$. Since $T \bar{x} \neq \emptyset$, $T \bar{x}=\{\bar{x}\}$.

Let $(X, d)$ be a cone metric space, and let $F: X \times X \rightarrow 2^{E}$ be a mapping. We define a relation $\triangleleft$ on $X$ as follows: for any $x, y \in X$,

$$
y \triangleleft x \text { if and only if } F(x, y)+d(x, y) \subset-P .
$$

For each $x \in X$, we denote $S(x)=\{y \in X: y \triangleleft x\}$.

Let $(X, d)$ be a cone metric space, and let $F: X \times X \rightarrow 2^{E}$ be a set valued mapping. Consider the following conditions, respectively.

(F1) $0 \in F(x, x)$ and $F(x, x) \subset-P$ for all $x \in X$.

(F2) $F(x, y) \subset F(x, z)+F(z, y)-P$ for all $x, y, z \in X$.

(F3) $F(x, \cdot)$ is bounded from below for each $x \in X$.

(F4) $F(x, \cdot)$ is sequentially lower semi-continuous for each $x \in X$.

Lemma 3.2. Let $(X, d)$ be a cone metric space such that $P$ is regular. Suppose that a set valued mapping $F: X \times X \rightarrow 2^{E}$ satisfies (F1), (F2), (F3) and (F4).

If $\triangleleft$ is a relation defined as (3.1), then we have the followings.

(a) $\triangleleft$ is a preodering on $X$;

(b) For each $x \in X, S(x)$ is sequentially closed in $(X, d)$;

(c) $S(y) \subset S(x)$ whenever $y \in S(x)$ for $x, y \in X$;

(d) For each $x \in X,\{d(x, y): y \in S(x)\}$ is bounded;

(e) If $x_{n+1} \triangleleft x_{n}$ for all $n \geq 0$, then $\sum_{n=0}^{\infty} d\left(x_{n}, x_{n+1}\right)$ is convergent. 
Proof. Let $\triangleleft$ be the relation defined as (3.1). Then from $(F 1) x \triangleleft x$ for each $x \in X$. Let $x \triangleleft y$ and $y \triangleleft z$ for $x, y, z \in X$. Then $F(y, x)+d(y, x) \subset-P$ and $F(z, y)+d(z, y) \subset-P$.

Since $F(z, x) \subset F(z, y)+F(y, x)-P$, we obtain

$$
\begin{aligned}
F(z, x)+d(y, x)+d(y, z) & \subset F(z, y)+F(y, x)+d(y, x)+d(y, z)-P \\
& \subset-P .
\end{aligned}
$$

Because $d(x, z)-d(y, x)-d(y, z) \in-P, F(z, x)+d(z, x) \subset-P$. Thus $x \triangleleft z$. Hence (a) is proved.

For each $x \in X$, since $d(x, \cdot)+F(x, \cdot)$ is sequentially lower semi-continuous, $S(x)$ is sequentially closed, and so (b) is proved.

Let $y \in S(x)$ and $z \in S(y)$. Then $y \triangleleft x$ and $z \triangleleft y$. Thus we have $z \triangleleft x$, and so $z \in S(x)$. Hence $S(y) \subset S(x)$, and (c) is proved.

Let $x \in X$ and $y \in S(x)$. Then $y \triangleleft x$, and so $F(x, y)+d(x, y) \subset-P$. Hence $d(x, y) \in-F(x, y)+(-P)$. By (F3), $F(x, y)$ is bounded from below. Thus there exists $z \in E$ such that $F(x, y)-z \subset P$. Thus $F(x, y) \subset z+P$. Hence $d(x, y) \in-z-P$, and hence $0 \leq d(x, y) \leq-z$. Thus $\{d(x, y): y \in S(x)\}$ is bounded.

Finally, we show that (e) is satisfied.

Let $x_{n+1} \triangleleft x_{n}$ for all $n \geq 0$. Then $F\left(x_{k}, x_{k+1}\right)+d\left(x_{k}, x_{k+1}\right) \subset-P$, and so $\sum_{k=1}^{n} F\left(x_{k}, x_{k+1}\right)+\sum_{k=1}^{n} d\left(x_{k}, x_{k+1}\right) \subset-P$. By (F2), we have $F\left(x_{1}, x_{n+1}\right) \subset$ $\sum_{k=1}^{n} F\left(x_{k}, x_{k+1}\right)-P$. Thus we obtain $F\left(x_{1}, x_{n+1}\right) \subset-\sum_{k=1}^{n} d\left(x_{k}, x_{k+1}\right)-$ $P$, and so $\sum_{k=1}^{n} d\left(x_{k}, x_{k+1}\right) \subset-F\left(x_{1}, x_{n+1}\right)-P$. From (F3) there exists $z \in E$ such that $F\left(x_{1}, x_{n+1}\right)-z \subset P$. Hence we have $\sum_{k=1}^{n} d\left(x_{k}, x_{k+1}\right) \in$ $-z-P$, and hence $\sum_{k=1}^{n} d\left(x_{k}, x_{k+1}\right) \leq-z$ for all $n \geq 1$. Since $P$ is regular, $\sum_{n=0}^{\infty} d\left(x_{n}, x_{n+1}\right)$ is convergent.

Theorem 3.1. Let $(X, d)$ be a complete cone metric space such that $P$ is complete and regular. Suppose that a set valued mapping $F: X \times X \rightarrow 2^{E}$ satisfies (F1), (F2), (F3) and (F4).

If $\triangleleft$ is a preodering defined as (3.1), then for each $x_{0} \in X$ there exists $\bar{x} \in X$ such that $\bar{x} \in S\left(x_{0}\right)$ and $S(\bar{x})=\{\bar{x}\}$.

Proof. Let $x_{0} \in X$ be fixed. We can choose $x_{1} \in S\left(x_{0}\right)$ by Lemma 3.2(d) such that

$$
\frac{1}{2} \sup \left\{\left\|d\left(x_{0}, u\right)\right\|: u \in S\left(x_{0}\right)\right\} \leq\left\|d\left(x_{0}, x_{1}\right)\right\| .
$$

Again, we choose $x_{2} \in S\left(x_{1}\right)$ such that

$$
\frac{1}{2} \sup \left\{\left\|d\left(x_{1}, v\right)\right\|: v \in S\left(x_{1}\right)\right\} \leq\left\|d\left(x_{1}, x_{2}\right)\right\| .
$$

Inductively, we can choose a sequence $\left\{x_{n}\right\}$ of points in $X$ such that

$$
x_{n+1} \in S\left(x_{n}\right) \text { and } \frac{1}{2} \sup \left\{\left\|d\left(x_{n}, w\right)\right\|: w \in S\left(x_{n}\right)\right\} \leq\left\|d\left(x_{n}, x_{n+1}\right)\right\|
$$

for all $n \geq 1$. 
From Lemma 3.2(c), we have $S\left(x_{n+1}\right) \subset S\left(x_{n}\right)$ for all $n \geq 0$. Hence condition (1) of Lemma 3.1 is satisfied.

From Lemma $3.2(\mathrm{e}), \sum_{k=1}^{\infty} d\left(x_{k}, x_{k+1}\right)$ is convergent. Thus, for any $c \in$ $\operatorname{int}(P)$, there exists $n_{0} \in \mathbb{N}$ such that $\sum_{k>n_{0}} d\left(x_{k}, x_{k+1}\right) \ll c$, and so $d\left(x_{n}, x_{m}\right)$ $\ll c$ for all $m>n>n_{0}$. Hence $\left\{x_{n}\right\}$ is a Cauchy sequence in $X$. Since $X$ is complete, there exists $\bar{x} \in X$ such that $\lim _{n \rightarrow \infty} x_{n}=\bar{x}$. From (3.2) we have $\lim \sup \left\{\left\|d\left(x_{n}, w\right)\right\|: w \in S\left(x_{n}\right)\right\}=0$. Hence condition (2) of Lemma 3.1 is satisfied.

From Lemma 3.2(b), $S\left(x_{n}\right)$ is sequentially closed for all $n \geq 0$. Since $\left\{x_{k+1}\right\}_{k \geq n} \subset S\left(x_{n}\right), \bar{x} \in S\left(x_{n}\right)$ for all $n \geq 0$. Hence $\bar{x} \in S\left(x_{0}\right)$.

From Lemma 3.2(c), $S(\bar{x}) \subset S\left(x_{n}\right)$ for all $n \geq 0$. Thus all conditions of Lemma 3.1 are satisfied, and from Lemma $3.1 S(\bar{x})=\{\bar{x}\}$.

Theorem 3.2. Let $(X, d)$ be a complete cone metric space such that $P$ is complete and regular. Suppose that a set valued mapping $F: X \times X \rightarrow 2^{E}$ satisfies (F1), (F2), (F3) and (F4).

Assume that a set valued mapping $T: X \rightarrow 2^{X}$ satisfies the following condition: for each $x \in X$, there exists $y \in T x$ such that

$$
F(x, y)+d(x, y) \subset-P .
$$

Then $T$ has a fixed point in $X$.

Proof. We define a preordering $\triangleleft$ as (3.1). From Theorem 3.1 there exists $\bar{x} \in X$ such that $S(\bar{x})=\{\bar{x}\}$. From (3.3), there exists $z \in T \bar{x}$ such that $z \triangleleft \bar{x}$. Hence $z \in S(\bar{x})$, and hence $z=\bar{x}$. Thus, $\bar{x} \in T \bar{x}$.

Remark 3.1. If $d$ is a complete metric (resp. cone metric) on a set $X$, then for each $\epsilon>0, \epsilon d$ is also a complete metric (resp. cone metric) on a set $X$.

From Remark 3.1 and Theorem 3.2 we have the following result.

Corollary 3.3. Let $(X, d)$ be a complete cone metric space such that $P$ is complete and regular. Suppose that a set valued mapping $F: X \times X \rightarrow 2^{E}$ satisfies (F1), (F2), (F3) and (F4).

Assume that a set valued mapping $T: X \rightarrow 2^{X}$ satisfies the following condition: for each $\epsilon>0$ and for each $x \in X$, there exists $y \in T x$ such that

$$
F(x, y)+\epsilon d(x, y) \subset-P .
$$

Then $T$ has a fixed point in $X$.

From Theorem 3.2 and Corollary 3.3 we obtain the following two corollaries, respectively.

Corollary 3.4. Let $(X, d)$ be a complete cone metric space such that $P$ is complete and regular. Suppose that a set valued mapping $F: X \times X \rightarrow 2^{E}$ satisfies (F1), (F2), (F3) and (F4).

Assume that a mapping $f: X \rightarrow X$ satisfies the following condition:

$$
F(x, f x)+d(x, f x) \subset-P
$$


for each $x \in X$. Then $f$ has a fixed point in $X$.

Corollary 3.5. Let $(X, d)$ be a complete cone metric space such that $P$ is complete and regular. Suppose that a set valued mapping $F: X \times X \rightarrow 2^{E}$ satisfies (F1), (F2), (F3) and (F4).

Assume that a mapping $f: X \rightarrow X$ satisfies the following condition:

$$
F(x, f x)+\epsilon d(x, f x) \subset-P
$$

for each $\epsilon>0$ and for each $x \in X$. Then $f$ has a fixed point in $X$.

Remark 3.2. Let $(X, \rho)$ be a complete metric space and $e \in \operatorname{int}(P)$ be fixed. Then $\rho(\cdot, \cdot) e$ is a complete cone metric on $X$. Also, $K=\{r e: r \geq 0\}$ is a complete and regular cone.

From Remark 3.2 and Theorem 3.2 we obtain the following results.

Corollary 3.6. Let $(X, \rho)$ be a complete metric space. Suppose that a set valued mapping $F: X \times X \rightarrow 2^{E}$ satisfies (F1), (F2), (F3) and (F4).

Assume that a set valued mapping $T: X \rightarrow 2^{X}$ satisfies the following condition: for each $x \in X$, there exists $y \in T x$ such that

$$
F(x, y)+\rho(x, y) e \subset-P .
$$

Then $T$ has a fixed point in $X$.

\section{Ekeland type variational principle}

In this section, we give an Ekeland's variational principle for set-valued mappings, which is closely realated with generalized equilibrium problems.

Theorem 4.1. Let $(X, d)$ be a complete cone metric space such that $P$ is complete and regular. Suppose that a set valued mapping $F: X \times X \rightarrow 2^{E}$ satisfies (F1), (F2), (F3) and (F4). Then, for every $x_{0} \in X$, there exists $\bar{x} \in X$ such that

(a) $F\left(x_{0}, \bar{x}\right)+d\left(x_{0}, \bar{x}\right) \subset-P$,

(b) $F(\bar{x}, x)+d(\bar{x}, x) \not \subset-P$ for all $x \neq \bar{x}$.

Proof. Let $\triangleleft$ be a preodering defined as (3.1), and let $x_{0} \in X$. Then from Theorem 3.1 there exists $\bar{x} \in X$ such that $\bar{x} \in S\left(x_{0}\right)$ and $S(\bar{x})=\{\bar{x}\}$.

Since $\bar{x} \in S\left(x_{0}\right)$, from (3.1) we obtain

$$
F\left(x_{0}, \bar{x}\right)+d\left(x_{0}, \bar{x}\right) \subset-P .
$$

For all $x \neq \bar{x}, x \notin S(\bar{x})$. Thus we have

$$
F(\bar{x}, x)+d(\bar{x}, x) \not \subset-P .
$$

Corollary 4.2. Let $(X, d)$ be a complete cone metric space such that $P$ is complete and regular. Suppose that a set valued mapping $F: X \times X \rightarrow 2^{E}$ satisfies (F1), (F2), (F3) and (F4). Then, for each $\epsilon>0$ and for every $x_{0} \in X$, there exists $\bar{x} \in X$ such that 
(a) $F\left(x_{0}, \bar{x}\right)+\epsilon d\left(x_{0}, \bar{x}\right) \subset-P$,

(b) $F(\bar{x}, x)+\epsilon d(\bar{x}, x) \not \subset-P$ for all $x \neq \bar{x}$.

Theorem 4.3. Let $(X, d)$ be a complete cone metric space such that $P$ is complete and regular. Suppose that a set valued mapping $F: X \times X \rightarrow 2^{E}$ satisfies (F1), (F2), (F3) and (F4). Then the following are equivalent.

(a) Ekeland type variational principle: For every $x_{0} \in X$, there exists $\bar{x} \in X$ such that $\bar{x} \in S_{0}=\left\{x \in X: F\left(x_{0}, x\right)+d\left(x_{0}, x\right) \subset-P\right\}$ and $F(\bar{x}, x)+d(\bar{x}, x) \not \subset$ $-P$ for all $x \neq \bar{x}$.

(b) Caristi-Kirk type fixed point theorem: Let $T: X \rightarrow 2^{X}$ be a set-valued mapping such that, for every $x_{*} \in S_{0}$, there exists $x \in T x_{*}$ satisfying $F\left(x_{*}, x\right)+$ $d\left(x_{*}, x\right) \subset-P$. Then there exists $\bar{x} \in S_{0}$ such that $\bar{x} \in T \bar{x}$.

(c) Oettli and Théra type theorem: Let $D \subset X$ such that, for every $x_{*} \in$ $S_{0} \backslash D$, there exists $x \in X$ such that $x \neq x_{*}$ and $F\left(x_{*}, x\right)+d\left(x_{*}, x\right) \subset-P$. Then there exists $\bar{x} \in S_{0} \cap D$.

Proof. (a) $\Rightarrow$ (c): Suppose that (a) and the hypothesis of (c) are satisfied.

Suppose that $S_{0} \cap D=\emptyset$. By (a), there exists $\bar{x} \in S_{0}$ and $F(\bar{x}, x)+d(\bar{x}, x) \not \subset$ $-P$ for all $x \neq \bar{x}$. Since $S_{0} \cap D=\emptyset, \bar{x} \in S_{0} \backslash D$. By the hypothesis of (c), there exists $x \in X$ such that $x \neq \bar{x}$ and $F(\bar{x}, x)+d(\bar{x}, x) \subset-P$, which is a contradiction.

(c) $\Rightarrow(\mathrm{a})$ : Assume that (c) is satisfied. For every $x_{0} \in X$, let $G\left(x_{0}\right)=\{x \in$ $\left.X: F\left(x_{0}, x\right)+d\left(x_{0}, x\right) \subset-P, x \neq x_{0}\right\}$. Let $D=\left\{x_{0}: G\left(x_{0}\right)=\emptyset\right\}$. If $x_{0} \notin D$, then $G\left(x_{0}\right) \neq \emptyset$. Hence there exists $x \in G\left(x_{0}\right)$, and so $x \neq x_{0}$ and $F\left(x_{0}, x\right)+d\left(x_{0}, x\right) \subset-P$. Thus the hypothesis of (c) is satisfied. By (c), there exists $\bar{x} \in S_{0} \cap D$. Thus $\bar{x} \in S_{0}$ and $F(\bar{x}, x)+d(\bar{x}, x) \not \subset-P$ for all $x \neq \bar{x}$.

(b) $\Rightarrow$ (c): Suppose that (b) and the hypothesis of (c) are satisfied.

Let $T x_{*}=\left\{x \in X: x \neq x_{*}\right\}$. Suppose that $S_{0} \cap D=\emptyset$. Then for all $x \in S_{0}$, $x \notin D$. By the hypothesis of (c), for every $x_{*} \in S_{0} \backslash D$, there exists $x \in X$ such that $x \neq x_{*}$ and $F\left(x_{*}, x\right)+d\left(x_{*}, x\right) \subset-P$. That is, for all $x_{*} \in S_{0}$, there exists $x \in X$ such that $x \in T x_{*}$ and $F\left(x_{*}, x\right)+d\left(x_{*}, x\right) \subset-P$. By (b), there exists $\bar{x} \in S_{0}$ such that $\bar{x} \in T \bar{x}$, and hence $\bar{x} \neq \bar{x}$, which is a contradiction.

(c) $\Rightarrow(\mathrm{b})$ : Suppose that (c) and the hypothesis of (b) are satisfied.

Suppose that $x \notin T x$ for all $x \in X$. Let $D=\{x \in X: x \in T x\}$. Then $D=\emptyset$. By the hypothesis of $(b)$, for every $x_{*} \in S_{0}$, there exists $x \in T x_{*}$ satisfying $F\left(x_{*}, x\right)+d\left(x_{*}, x\right) \subset-P$. Since $D=\emptyset, x_{*} \in S_{0} \backslash D$, and $x \neq x_{*}$. By (c), there exists $\bar{x} \in S_{0} \cap D$. Hence $D \neq \emptyset$, which is a contradiction.

From Remark 3.2 and Theorem 4.1 we have the following result.

Corollary 4.4. Let $(X, \rho)$ be a complete metric space. Suppose that a set valued mapping $F: X \times X \rightarrow 2^{E}$ satisfies (F1), (F2), (F3) and (F4). Then, for every $x_{0} \in X$, there exists $\bar{x} \in X$ such that

(a) $F\left(x_{0}, \bar{x}\right)+\rho\left(x_{0}, \bar{x}\right) e \subset-K$,

(b) $F(\bar{x}, x)+\rho(\bar{x}, x) e \not \subset-K$ for all $x \neq \bar{x}$.

From Remark 3.1 and Corollary 4.4 we obtain the following result. 
Corollary $4.5([33])$. Let $(X, \rho)$ be a complete metric space. Suppose that a set valued mapping $F: X \times X \rightarrow 2^{E}$ satisfies (F1), (F2), (F3) and (F4). Then, for each $\epsilon>0$ and for every $x_{0} \in X$, there exists $\bar{x} \in X$ such that

(a) $F\left(x_{0}, \bar{x}\right)+\epsilon \rho\left(x_{0}, \bar{x}\right) e \subset-K$,

(b) $F(\bar{x}, x)+\epsilon \rho(\bar{x}, x) e \not \subset-K$ for all $x \neq \bar{x}$.

\section{Equilibrium problems}

Let $K$ be a non-empty subset of a cone metric space $(X, d)$, and let $F$ : $K \times K \rightarrow 2^{E}$ be a mapping. By an equilibrium problem, we understand the problem of finding $\bar{x} \in K$ such that

$$
F(\bar{x}, x) \subset P \text { for all } x \in K .
$$

We consider some possible cases of equilibrium problems as follows: the problem of finding $\bar{x} \in K$ such that

$$
F(\bar{x}, x) \subset E-\operatorname{int}(-P) \text { for all } x \in K,
$$

the problem of finding $\bar{x} \in K$ such that

$$
F(\bar{x}, x) \not \subset \operatorname{int}(-P) \text { for all } x \in K,
$$

the problem of finding $\bar{x} \in K$ such that

$$
F(\bar{x}, x) \cap(E-\operatorname{int}(-P)) \neq \emptyset \text { for all } x \in K \text {. }
$$

Note that $\bar{x}$ is a solution of $(5.1) \Rightarrow \bar{x}$ is a solution of $(5.2) \Rightarrow \bar{x}$ is a solution of $(5.3) \Leftrightarrow \bar{x}$ is a solution of $(5.4)$.

Let $K$ be a non-empty subset of a cone metric space $(X, d)$, and let $F$ : $K \times K \rightarrow 2^{E}$ be a mapping and $\epsilon>0$. A point $\bar{x} \in K$ is called an $\epsilon$-solution of equilibrium problem (5.1) if

$$
F(\bar{x}, x)+\epsilon d(\bar{x}, x) \subset P
$$

for all $x \in K$.

Without any convexity assumption, we study on existence theorems of solution for equilibrium problem (5.1) on cone metric spaces.

Theorem 5.1. Let $(X, d)$ be a cone metric space, and let $K$ be a sequentially compact subset of $X$. Suppose that a mapping $F: K \times K \rightarrow 2^{E}$ satisfies the following conditions:

(1) for each $y \in K, F(\cdot, y)$ is sequentially lower semi-continuous.

(2) for each $\epsilon>0$, there exists an $\epsilon$-solution $x \in K$ of equilibrium problem (5.1). That is, there exists $x \in K$ such that

$$
F(x, y)+\epsilon d(x, y) \subset P
$$

for all $y \in K$. Then there exists a solution $\bar{x} \in K$ of equilibrium problem (5.1). 
Proof. By (2), for each $n \in \mathbb{N}$, there exists $x_{n} \in K$ such that

$$
F\left(x_{n}, y\right)+\frac{1}{n} d\left(x_{n}, y\right) \subset P
$$

for all $y \in X$.

Since $K$ is sequentially compact, we may assume that there exists $\bar{x} \in K$ such that $\lim _{n \rightarrow \infty} x_{n}=\bar{x}$.

For fixed $y \in K$ and for fixed $n \in \mathbb{N}$, let $L_{n, y}(P)=\{x \in K: F(x, y)+$ $\left.\frac{1}{n} d(x, y) \subset P\right\}$. By Lemma 2.3 and Lemma $2.5, F(\cdot, y)+\frac{1}{n} d(\cdot, y)$ is sequentially lower semi-continuous. By applying Lemma $2.1, L_{n, y}(P)$ is sequentially closed.

For $m>n$, we obtain

$F\left(x_{m}, y\right)+\frac{1}{n} d\left(x_{m}, y\right)=F\left(x_{m}, y\right)+\frac{1}{m} d\left(x_{m}, y\right)+\left(\frac{1}{n}-\frac{1}{m}\right) d\left(x_{m}, y\right) \subset P+P=P$.

Thus, $\left\{x_{m}\right\}_{m>n} \subset L_{n, y}(P)$, and so $\bar{x} \in L_{n, y}(P)$ for all $y \in K$ and for all $n \in \mathbb{N}$. Hence $F(\bar{x}, y)+\frac{1}{n} d(\bar{x}, y) \subset P$.

By letting $n \rightarrow \infty$ in above inequality, we have $F(\bar{x}, y) \subset P$ for all $y \in K$.

Let $(X, d)$ be a cone metric space. We say that $x_{0} \in X$ satisfies property (B.C) if every sequence $\left\{x_{n}\right\} \subset X$ satisfying $F\left(x_{0}, x_{n}\right) \subset-P$ for all $n \in \mathbb{N}$ and $F\left(x_{n}, x\right)+\frac{1}{n} d\left(x_{n}, x\right) \not \subset-P$ for all $x \in X$ and for all $n \in \mathbb{N}$, has a convergent subsequence.

Theorem 5.2. Let $(X, d)$ be a complete cone metric space such that $P$ is complete and regular. Suppose that a set valued mapping $F: X \times X \rightarrow K(E)$ satisfies $(\mathrm{F} 1),(\mathrm{F} 2),(\mathrm{F} 3)$ and $(\mathrm{F} 4)$, and $F(\cdot, y)$ is sequentially upper semicontinuous for each $y \in X$. If some $x_{0} \in X$ satisfies property (B.C), then equilibrium problem (5.4) has a solution.

Proof. Let $\epsilon=\frac{1}{n}$ in Corollary 4.2. Then for each $n \in \mathbb{N}$ and for each $x_{0} \in X$, there exists $x_{n} \in X$ such that $F\left(x_{0}, x_{n}\right)+\frac{1}{n} d\left(x_{0}, x_{n}\right) \subset-P$ and $F\left(x_{n}, x\right)+$ $\frac{1}{n} d\left(x_{n}, x\right) \not \subset-P$ for all $x \in X$.

Since $-\frac{1}{n} d\left(x_{0}, x_{n}\right) \in-P, F\left(x_{0}, x_{n}\right) \subset-P$ for all $n \in \mathbb{N}$. Hence $x_{0}$ is satisfying condition (B.C), and so there exists a subsequence $\left\{x_{n(k)}\right\}$ of $\left\{x_{n}\right\}$ such that $\lim _{k \rightarrow \infty} x_{n(k)}=\bar{x}$ for some $\bar{x} \in X$.

For fixed $y \in K$ and for fixed $n(k) \in \mathbb{N}$, let

$U_{n(k), y}(E-\operatorname{int}(-P))=\left\{x \in K: F(x, y)+\frac{1}{n(k)} d(x, y) \cap(E-i n t(-P)) \neq \emptyset\right\}$. By Lemma 2.4 and Lemma $2.5, F(\cdot, y)+\frac{1}{n(k)} d(\cdot, y)$ is sequentially upper semicontinuous. By applying Lemma 2.2, $U_{n(k), y}(E-\operatorname{int}(-P))$ is sequentially closed.

For $m(k)>n(k)$, since $F\left(x_{m(k)}, y\right)+\frac{1}{m(k)} d\left(x_{m(k)}, y\right) \not \subset-P$ and $-P-\left(\frac{1}{n(k)}-\right.$ $\left.\frac{1}{m(k)}\right) d\left(x_{m(k)}, y\right)=-P$, we obtain

$$
F\left(x_{m(k)}, y\right)+\frac{1}{n(k)} d\left(x_{m(k)}, y\right)
$$




$$
\begin{aligned}
& =F\left(x_{m(k)}, y\right)+\frac{1}{m(k)} d\left(x_{m(k)}, y\right)+\left(\frac{1}{n(k)}-\frac{1}{m(k)}\right) d\left(x_{m(k)}, y\right) \\
& \not \subset-P .
\end{aligned}
$$

Hence we have $F\left(x_{m(k)}, y\right)+\frac{1}{n(k)} d\left(x_{m(k)}, y\right) \not \subset i n t(-P)$, and hence,

$$
F\left(x_{m(k)}, y\right)+\frac{1}{n(k)} d\left(x_{m(k)}, y\right) \cap(E-i n t(-P)) \neq \emptyset .
$$

Thus, $\left\{x_{m(k)}\right\}_{m(k)>n(k)} \subset U_{n(k), y}(E-\operatorname{int}(-P))$. So $\bar{x} \in U_{n, y}(E-i n t(-P))$ for all $y \in K$. Hence $F(\bar{x}, y)+\frac{1}{n(k)} d(\bar{x}, y) \cap(E-\operatorname{int}(-P)) \neq \emptyset$.

By letting $k \rightarrow \infty$ in above inequality, we have $F(\bar{x}, y) \cap(E-\operatorname{int}(-P)) \neq \emptyset$ for all $y \in K$.

Theorem 5.3. Let $(X, d)$ be a complete cone metric space such that $P$ is complete and regular. Suppose that a set valued mapping $F: X \times X \rightarrow 2^{E}$ satisfies (F1), (F2), (F3) and (F4). For every $x_{0} \in X$, let $S_{0}=\{x \in X$ : $\left.F\left(x_{0}, x\right)+d\left(x_{0}, x\right) \subset-P\right\}$. Then the following are equivalent.

(a) Existence of solution for equilibrium problem: Assume that, for every $x_{*} \in S_{0}$, there exists $y \in X$ such that $y \neq x_{*}$ and $F\left(x_{*}, y\right)+d\left(x_{*}, y\right) \subset-P$. Then there exists $\bar{x} \in S_{0}$ such that $F(\bar{x}, x) \subset P$ for all $x \in X$.

(b) Oettli and Théra type theorem: Let $D \subset X$ such that, for every $x_{*} \in$ $S_{0} \backslash D$, there exists $x \in X$ such that $x \neq x_{*}$ and $F\left(x_{*}, x\right)+d\left(x_{*}, x\right) \subset-P$. Then there exists $\bar{x} \in S_{0} \cap D$.

Proof. $(\mathrm{a}) \Rightarrow(\mathrm{b})$ : Suppose that (a) and the hypothesis of (b) are satisfied. Then there exists $\bar{x} \in S_{0}$ such that $F(\bar{x}, x) \subset P$ for all $x \in X$.

Suppose that $S_{0} \cap D=\emptyset$. Then $\bar{x} \in S_{0} \backslash D$. By the hypothesis of (b), there exists $y \in X$ such that $y \neq \bar{x}$ and $F(\bar{x}, y)+d(\bar{x}, y) \subset-P$. Thus $F(\bar{x}, y) \subset-P$. Hence we have $F(\bar{x}, y)=\{0\}$. Hence $d(\bar{x}, y) \in-P$, and hence $\bar{x}=y$, which is a contradiction.

$(\mathrm{b}) \Rightarrow(\mathrm{a})$ : Suppose that (b) and the hypothesis of (a) are satisfied. Let $D=\{x \in X: F(x, y) \subset P$ for all $y \in X\}$. By the hypothesis of (a), for all $x_{*} \in S_{0} \backslash D$, there exists $x \in X$ such that $x \neq x_{*}$ and $F\left(x_{*}, x\right)+d\left(x_{*}, x\right) \subset-P$. By (b), there exists $\bar{x} \in S_{0} \cap D$. Hence $\bar{x} \in D$ and $F(\bar{x}, y) \subset P$ for all $y \in X$.

From Remark 3.2, Theorem 4.3 and Theorem 5.3 we have the following result.

Corollary 5.4. Let $(X, \rho)$ be a complete metric space. Suppose that a set valued mapping $F: X \times X \rightarrow 2^{E}$ satisfies (F1), (F2), (F3) and (F4). Then the following are equivalent.

(a) Ekeland type variational principle: For every $x_{0} \in X$, there exists $\bar{x} \in X$ such that $\bar{x} \in M_{0}=\left\{x \in X: F\left(x_{0}, x\right)+\rho\left(x_{0}, x\right) e \subset-K\right\}$ and $F(\bar{x}, x)+$ $\rho(\bar{x}, x) e \not \subset-K$ for all $x \neq \bar{x}$. 
(b) Caristi-Kirk type fixed point theorem: Let $T: X \rightarrow 2^{X}$ be a setvalued mapping such that, for every $x_{*} \in M_{0}$, there exists $x \in T x_{*}$ satisfying $F\left(x_{*}, x\right)+\rho\left(x_{*}, x\right) e \subset-K$. Then there exists $\bar{x} \in X$ such that $\bar{x} \in T \bar{x}$.

(c) Oettli and Théra type theorem: Let $D \subset X$ such that, for every $x_{*} \in$ $M_{0} \backslash D$, there exists $x \in X$ such that $x \neq x_{*}$ and $F\left(x_{*}, x\right)+\rho\left(x_{*}, x\right) e \subset-K$. Then there exists $\bar{x} \in M_{0} \cap D$.

(d) Existence of solution for equilibrium problem: Assume that, for every $x_{*} \in M_{0}$, there exists $y \in X$ such that $y \neq x_{*}$ and $F\left(x_{*}, y\right)+\rho\left(x_{*}, y\right) e \subset-K$. Then there exists $\bar{x} \in M_{0}$ such that $F(\bar{x}, x) \subset K$ for all $x \in X$.

\section{References}

[1] S. Al-Homidan, Q. H. Ansaria, and J. C. Yao, Some generalizations of Ekeland-type variational principle with applications to equilibrium problems and fixed point theory, Nonlinear Anal. 69 (2008), no. 1, 126-139.

[2] M. Abbas and G. Jungck, Common fixed point results for noncommuting mappings without continuity in cone metric spaces, J. Math. Anal. Appl. 341 (2008), no. 1, 416420.

[3] M. Abbas and B. E. Rhoades, Fixed and periodic point results in cone metric spaces, Applied Mathematics Letters (2008), doi:10.1016/j.akl.2008.07.001.

[4] M. Ashad, A. Azam, and P. Vetro, Some common fixed results in cone metric spaces, Fixed point theory and Apllications 2009, Article ID 493965, 11pages, 2009.

[5] J. P. Aubin and H. Frankowska, Set-Valued Analysis, Systems \& Control: Foundations \& Applications, 2. Birkhäuser Boston, Inc., Boston, MA, 1990.

[6] M. Bianchi, G. Kassay, and R. Pini, Existence of equilibria via Ekeland's principle, J. Math. Anal. Appl. 305 (2005), no. 2, 502-512.

[7] S. H. Cho and J. S. Bae, Common fixed point theorems for mappings satisfying property (E.A) on cone metric spaces, Math. Comput. Modelling 53 (2011), no. 5-6, 945-951.

[8] _ Fixed point theorems for multivalued maps in cone metric spaces, Fixed Point Theory Appl. 2011 (2011), 87, 7 pp.

[9] W. S. Du, A note on cone metric fixed point theory and its equivalence, Nonlinear Anal. 72 (2010), no. 5, 2259-2261.

[10] D. G. De Figueiredo, The Ekeland Variational Principle with Applications and Detours, Tata Institute of Fundamental Research, Bombay, 1989.

[11] I. Ekeland, Sur les problems variationnels, C. R. Acad. Sci. Par 275 (1972), 1057-1059.

[12] _ On the variational principle, J. Math. Anal. Appl. 47 (1974), 324-353.

[13] , Nonconvex minimization problems, Bull. Amer. Math. Soc. (N.S.) 1 (1979), no. $3,443-474$.

[14] L. G. Huang and X. Zhang, Cone metric spaces and fixed point theorems of contractive mappings, J. Math. Anal. Appl. 332 (2007), no. 2, 1468-1476.

[15] D. Ilić and V. Rakočević, Common fixed points for maps on cone metric spaces, J. Math. Anal. Appl. 341 (2008), no. 2, 876-882.

[16] _ Quasi-contraction on cone metric spaces, Applied Mathematics Letters (2008), doi:10.1016/j.aml.2008.08.001.

[17] S. Janković, Z. Kadelburg, S. Radenović, and B. E. Rhoades, Assad-Kirk-type fixed point theorems for a pair of nonself mappings on cone metric spaces, Fixed Point Theory Appl. 2009 (2009), Art. ID 761086, 16 pp.

[18] G. Jungck, S. Radenović, S. Radojević, and V. Rakočević, Common fixed point theorems for weakly compatible pairs on cone metric spaces, Fixed Point Theory Appl. 2009 (2009), Art. ID 643840, 13 pp. 
[19] Z. Kadelburg, M. Pavlović, and S. Radenović, Common fixed point theorems for ordered contractions and quasicontractions in ordered cone metric spaces, Comput. Math. Appl. 59 (2010), no. 9, 3148-3159.

[20] Z. Kadelburg, S. Radenović, and V. Rakočević, Remaks on Quasi-contraction on a cone metric spaces, Applied Math. Letters 22 (2009), 1674-1679.

[21] Z. Kadelburg, S. Radenović, and B. Rosić, Strict contractive conditions and common fixed point theorems in cone metric spaces, Fixed Point Theory Appl. 2009 (2009), Art. ID $173838,14 \mathrm{pp}$.

[22] O. Kada, T. Suzuki, and W. Takahashi, Nonconvex minimization theorems and fixed point theorems in complete metric spaces, Math. Japon. 44 (1996), no. 2, 381-391.

[23] D. Klim and D. Wardowski, Dynamic processes and fixed points of set-valued nonlinear contractions in cone metric spaces, Nonlinear Anal. 71 (2009), no. 11, 5170-5175.

[24] L. J. Lin and W. S. Du, Ekeland's variational principle, minimax theorems and existence of nonconvex equilibria in complete metric spaces, J. Math. Anal. Appl. 323 (2006), no. $1,360-370$.

[25] J. P. Penot, The drop theorem, the petal theorem and Ekeland's variational principle, Nonlinear Anal. 10 (1986), no. 9, 813-822.

[26] S. Radenović, Common fixed points under contractive conditions in cone metric spaces, Comput. Math. Appl. 58 (2009), no. 6, 1273-1278.

[27] Sh. Rezapour, R. Haghi, and N. Shahzad, Some notes on fixed points of quasicontraction maps, Appl. Math. Lett. 23 (2010), no. 4, 498-502.

[28] Sh. Rezapour and R. Hamlbarani, Some notes on the paper "Cone metric spaces and fixed point theorems of contractive mappings", J. Math. Anal. Appl. 345 (2008), no. 2, $719-724$.

[29] T. Suzuki, Generalized distance and existence theorems in complete metric spaces, J. Math. Anal. Appl. 253 (2001), no. 2, 440-458.

[30] W. Takahashi, Nonlinear Functional Analysis, Yokohama Publishers, Yokohama, Japan, 2000.

[31] K. Wlodarczyk, R. Plebaniak, and C. Obczyński, Convergence theorems, best approximation and best proximity for set-valued dynamic systems of relatively quasi-asymptotic contractions in cone uniform spaces, Nonlinear Anal. 72 (2010), no. 2, 794-805.

[32] S. K. Yang, J. S. Bae, and S. H. Cho, Coincidence and common fixed and periodic point theorems in cone metric spaces, Comput. Math. Appl. 61 (2011), no. 2, 170-177.

[33] J. Zeng and S. J. Li, An Ekeland's variational principle for set-valued mappings with applications, J. Comput. Appl. Math. 230 (2009), no. 2, 477-484.

[34] Z. Zhao and X. Chen, Fixed points of decreasing operators in ordered Banach spaces and applications to nonlinear second order elliptic equations, Comput. Math. Appl. 58 (2009), no. 6, 1223-1229.

\section{JONG-SOOK BAE}

DePARTMENT OF MATHEMATiCs

MOYNGJI UNIVERSITY

YONGIN 449-728, KOREA

E-mail address: jsbae@mju.ac.kr

SEONG-HoOn CHO

Department of Mathematics

HANSEO UNIVERSITY

Chungnam 356-706, Korea

E-mail address: shcho@hanseo.ac.kr 